\title{
Las relaciones públicas: una actividad emergente al servicio de la sociedad de la información
}

\author{
Pedro Antonio Rojo Villada *
}

\begin{abstract}
Resumen
Parece que todo va a cambiar, y de hecho todo esta cambiando en las relaciones que mantienen las organizaciones con los medios de comunicación. Las organizaciones son entes complejos y a la vez dinámicos, dentro de un mundo cada vez más cualificado y donde las necesidades de contacto y de relación son mayores. El valor de una organización ya no se encuentra tanto en sus realizaciones materiales sino más bien en aquellos valores que es capaz de transmitir al mercado. La sociedad demanda toda una amplia gama de tipos de informaciones y comunicaciones, incluso a sabiendas de que, tras la propia información se encuentran intereses particulares, empresariales, políticos y económicos. Esta voracidad de informaciones y comunicaciones también encuentra eco en los medios de comunicación, más proclives si cabe a la inclusión de informaciones especializadas, que a la inclusión de informaciones generalistas, consideradas rutinarias.

Descriptores: Relaciones Públicas; Sociedad de la Información; Periodismo Especializado; Opinión Pública; Persuasión; Públicos; Comunicación Corporativa; Segmentación; posicionamiento.
\end{abstract}

\section{Introducción}

El momento apasionante que esta viviendo el mundo de la comunicación en nuestra época, así como las actividades comunicativas que se originan en el seno de las empresas e instituciones han contribuido a crear grandes expectativas sobre el papel que han de desempeñar los comunicadores en las organizaciones. A medida que la sociedad va transformando paulatinamente sus actividades de producción-consumo

\footnotetext{
* Dr. Pedro Antonio Rojo Villada. Profesor Agregado de la Facultad de Ciencias de la Comunicación Universidad Católica San Antonio de Murcia (España)

Correo-e: parojo@pdi.ucam.edu
}

Comun. Inf., v. 6, n. 1, p.30-45, jan./jun. 2003 
de bienes y servicios materiales en producción-consumo de bienes de información, la labor a desempeñar por los profesionales de las Relaciones Públicas comienza a ser cada vez más urgente'. De esta manera, las organizaciones perciben la necesidad de que las Relaciones Públicas abandonen el cordón umbilical que le unían al Marketing y que vayan ocupando un lugar cada vez más preponderante en la nueva economía basas en el consumo y en el intercambio de bienes intangibles (informaciones).

Esta liberalización, que coincide cronológicamente con el boom de Internet y las tecnologías digitales, y que camina a la par de la transformación económica de nuestras sociedades en sociedades consumidoras de información y de bienes intangibles (lo que se denomina en la actualidad, como "nueva economía"), en donde el sector terciario acapara todo el protagonismo, decimos que esta liberalización también va a afectar a la configuración profesional del Responsable de Relaciones Públicas. Ya quedaron muy lejos los tiempos del amateur, y del agente de prensa salido de las redacciones de los medios. Los nuevos tiempos ya reclaman un profesional con profundos conocimientos humanísticos (para crear sensibilidad en el público) y técnicos (para comunicar eficazmente la información de la organización a la que representa). A su vez, las funciones del Relaciones Públicas tradicional, cuyo cometido era la gestión de actividades de cara a los medios de comunicación, va a sufrir un cambio tanto cuantitativo como cualitativo. A este profesional se le van a atribuir nuevas responsabilidades en la gestión de la comunicación de su organización, y poco a poco ira ganado nuevas parcelas: comunicación interna, patrocinios, identidad corporativa, etc.

Los cambios revolucionarios de los últimos años no solo ha afectado a sus funciones, sino que también han sacudido a su denominación: Relaciones Públicas, Relaciones Externas, Relaciones Institucionales, Director de Comunicación, Jefe de prensa, Responsable de imagen, etc. términos que ha aportado mayor confusión, si cabe, a la hora de clarificar el papel desempeñado por este profesional en el seno de las organizaciones.

\section{Diversidad de mercados de la información}

La importancia adquirida por la información en los procesos de intercambio económicos llevo a la constitución de entidades jurídicas

Comun. Inf., v. 6, n. 1, p.30-45, jan./jun. 2003 
dedicadas exclusivamente a la actividad informativa o actividad empresarial de informar. Estas entidades colectivas elaboraban informaciones para que fueran utilizadas por los diferentes actores de la economía de los países, y además servía de instrumento para el intercambio de conocimientos entre estos actores constitutivos de la oferta y demanda de bienes y servicios. Las entidades colectivas que asumían como objeto social la tarea de informar han sido denominadas empresas informativas, que convirtieron su propio objeto social, la actividad informativa, en una actividad económica prospera cuya sede fue el mercado de la información, como lugar de intercambio de productos informativos entre empresas informativas y consumidores de medios de comunicación.

Las actividades informativas desarrolladas en el mercado de la información, centradas en el producto informativo como bien o servicio a intercambiar en dicho mercado, obedecen a una doble naturaleza:

a) actividades promotoras de información: consisten en el intercambio informativo con la finalidad de estimular y buscar una respuesta en los receptores (comunicación persuasiva).

b) Actividades ejecutivas de información: estas consisten en el intercambio informativo tendente a satisfacer las necesidades de información y conocimiento de los receptores (comunicación informativa).

\section{Una profesión liberal al servicio de todos los públicos}

El boom de las publicaciones de carácter especializado (prensa económica, tecnológica, cultural) y la inclusión, dentro de las secciones de los medios tradicionales (prensa, radio y televisión) de mayores contenidos especializados, ha provocado que las informaciones especializadas, procedentes de las organizaciones, cobren cada vez mayor preponderancia, no solo a nivel informativo, sino también a nivel económico y social.

A su vez, el público consumidor de medios de comunicación demanda una información cada vez más exigente, analítica y de mayor calidad, alejada de los contenidos generalistas. Este consumidor reclama con insistencia estar mejor informado sobre los sectores que intervienen en la vida económica y cultural del país, pero con un lenguaje sencillo y coloquial, haciendo "próximos" para el público de los medios, aquellos

Comun. Inf., v. 6, n. 1, p.30-45, jan./jun. 2003 
contenidos especializados, con un lenguaje coloquial. este boom de la información económica y de las informaciones especializadas también ha favorecido un incremento espectacular de las relaciones entre las empresas y los medios de comunicación.

La empresa se ha percatado de lo beneficiosa que resulta una política informativa de puertas abiertas y de transparencia informativa, con las ventajas que representa la información en términos de credibilidad. Esta mayor sensibilidad, por parte de las organizaciones se ha consolidado, en los últimos años, como un fundamento esencial de algunas políticas empresariales, en términos de inversión en acciones de comunicación. La actividad económica, se quiera o no, forma parte del ámbito de lo público, y por tanto será objeto de demanda por parte de la sociedad. Además, los textos jurídicos reconocen el derecho del público a ser informado sobre las actividades de las organizaciones y el deber de estas, de facilitar información y de ser transparentes hacia la sociedad.

Así pues, los medios de comunicación social siempre generan una imagen de la organización, unas veces por acción y otras por omisión. Esta imagen puede ser modificada, reestructurada o canalizada a través del establecimiento eficaz de una estrategia de relaciones con la prensa. También hemos apuntado la necesidad que tienen los medios de comunicación de mantener vivo el interés de sus lectores, oyentes o telespectadores, así como de ampliar sus fuentes informativas y diversificar sus temas con contenidos de carácter especializado. Esta actividad mediática no es más que la satisfacción de un deseo demandado desde el público de los diferentes medios.

Al comienzo de esta introducción habíamos definido a la organización como un ente complejo y dinámico. Es necesario añadir, para ser sinceros, que no existe una organización estándar de las relaciones con la prensa en las organizaciones, aunque todas ellas, más o menos, realizan funciones similares o parecidas en dicha faceta. A la unificación de las practicas profesionales están contribuyendo eficazmente los profesionales egresados de las Facultades de Ciencias de la Comunicación, así como un importante elenco de textos que llegan actualmente a las librerías y que contribuyen a la reflexión y la consolidación de una practica profesional, la de las Relaciones Públicas. A los primeros, los profesionales, tanto los egresados de las facultades de Ciencias de la Comunicación como los que ya están a punto de serlo, va dirigida esta obra, como fruto de la experiencia vivida por su

Comun. Inf., v. 6, n. 1, p.30-45, jan./jun. 2003 
autor, de su reflexión, de la aportación de otros autores, del intercambio de ideas con sus colegas, y de sus anhelos y esperanzas por contribuir decididamente a consolidar el mundo de las Relaciones Públicas en nuestro país.

\section{¿Por que debe informar la organización?}

Las necesidades actuales de una organización, respecto al desarrollo de una adecuada política de relaciones con la prensa se deben canalizar a través de unos servicios planificados, que utilizan como exponente más relevante los medios de comunicación. Las organizaciones modernas utilizan los medios de comunicación como soportes de información controlada, dirigidas fundamentalmente a:

- Clientes.

- Empleados.

- Proveedores y colaboradores.

- Instituciones.

- Accionistas.

En esta tupida red de relaciones con sus públicos, la organización que emplea los medios de comunicación para emitir sus informaciones e propone conseguir una serie de objetivos, más o menos definidos, y que vendrían a coincidir con los siguientes:

Reforzar la imagen, notoriedad y prestigio de la organización, sus productos y sus marcas.

1. Comunicar a sus diferentes públicos mensajes e información interesante, propia de su actividad y entorno empresarial.

2. Ofrecer puntos de vista, realizaciones y filosofia de la organización respecto de un tema.

3. Dar a conocer, en su sentido más amplio, las actividades desarrolladas por la organización.

4. Satisfacer el derecho que tiene la sociedad a conocer la realidad de la organización.

Para Piñuel y Westphalen, las relaciones con la prensa deben ser de doble sentido: de una empresa hacia la prensa y viceversa ${ }^{2}$. Para cualquier organización, ya sea esta un ente económico o institucional sin animo de lucro, lo interesante de este tipo de contactos es el

Comun. Inf., v. 6, n. 1, p.30-45, jan./jun. 2003 
mantenimiento de unas relaciones eficaces y positivas con la prensa, y siempre marcadas por unos objetivos estructurales o permanentes:

- Aumentar la notoriedad de la organización.

- Transmitir una imagen definida en la estrategia de comunicación, o al menos, una imagen conforme a la deseada por la empresa.

- Favorecer un juicio positivo hacia la empresa, o al menos, suscitar un movimiento de simpatía, de comprensión.

Estos dos autores también citan la necesidad de cumplir, en las relaciones con la prensa unos objetivos coyunturales:

- Difundir toda información útil al público en lo que concierne a la empresa.

- Transmitir toda la información concerniente a la empresa y que este ligada a una actualidad de interés general ${ }^{3}$.

Estos autores incluyen como premisa de las relaciones reciprocas entre la organización y los medios de comunicación, la función de vigilancia, mediante acciones de seguimiento o monitoring de la prensa. Para una organización es muy importante conocer todo lo que la prensa. cuenta de ella, ya que este conocimiento le proporcionara pistas sobre el estado de su imagen en la sociedad. Además, el control de los contenidos informativos también proporcionara a la organización el conocimiento suficiente sobre el entorno que rodea su actividad económica: evolución del entorno económico, legislación, innovaciones tecnológicas, estado de la competencia, etc.

La comunicaciones algo sustancial e imprescindible para la empresa, por lo que su gestión, de cara a los medios de comunicación, exige que haya de plantearse, necesariamente, una política de comunicación adecuada a sus objetivos y eficaz, utilizando todos los medios de comunicación que la organización dispone en su entorno.

La empresa tiene una necesidad de comunicación con su entorno, por el puro y simple motivo de que necesita influir sobre el mismo. La empresa no es más que un ente con personalidad jurídica que pretende conseguir de otras personas, naturales o jurídicas, la simpatía para el favorecimiento de sus actividades. Las personas ajenas a la empresa, naturales o jurídicas, son muy heterogéneas en cuanto a necesidades, actitudes, comportamientos y preferencias. La empresa necesita

Comun. Inf., v. 6, n. 1, p.30-45, jan./jun. 2003 
comunicarse con ellas, intercambiar mensajes y conocimientos para desarrollar su actividad económica. Las relaciones con la prensa son, pues, un conjunto de actividades cuyo objetivo fundamental es "congraciarse" con el cuarto poder ${ }^{4}$, a fin de que en sus contenidos informativos, favorezcan la valoración de la empresa a efectos de una consolidación de una determinada imagen deseada, lógicamente hablamos de imagen positiva.

Los medios de comunicación representan para la empresa el ámbito propio de intermediación con sus públicos externos. Sin embargo no se trata de un soporte controlado por la empresa, ya que los competidores también aparecen en los medios, junto a los lideres de opinión, ajenos completamente a la empresa, y por ende, no controlados por esta, y que sin embargo, también juegan un papel destacado sobre las preferencias y deseos de los públicos externas.

Los medios de comunicación se convierten en el territorio propicio para este doble juego, o si se prefiere, doble papel, primero como instrumentos para la emisión de los mensajes e imagen de la empresa, y segundo, como jueces de la empresa, y como tales, pueden dirigir mensajes o manifestaciones proclives o contrarias a los intereses de la organización.

Lo cierto es que la carga de connotación de los mensajes, en el caso de los medios de comunicación, es mucho más fuerte que en otros soportes de comunicación masiva. En los medios de comunicación, las informaciones no solo son importantes desde el punto de vista del contenido, sino también desde su posicionamiento en el entramado del medio. la comunicación trasciende al puro mensaje formal para insertarse en un entorno cargado de significación: la colocación en la pagina, el cuerpo de las letras, o la foto elegida, en la prensa, o bien la carga de afectividad del presentador, en los medios audiovisuales, en determinadas ocasiones suele resultar más importante, en términos de empatía para el público de los medios, que el propio contenido de la noticia. En ese juego simbólico es donde el medio tiene absoluta libertad de actuación, influyendo en la imagen positiva o negativa de la empresa.

La necesidad de informar, por parte de la empresa, se ha de entender desde el papel que juega esta como un sistema en relación con otros sistemas o supersistemas, lo que nos conduce a considerar una serie de principios:

Comun. Inf., v. 6, n. 1, p.30-45, jan./jun. 2003 
1. La empresa, como unidad económica de producción, esta formada por una serie de partes o subsistemas: de producción, de distribución, de información, etc.

2. La empresa, a su vez, solamente existe como parte de un supersistema, formado por su entorno, y que a su vez se compone de otros subsistemas: político, socio-económico, jurídico, etc.

3. La empresa, como todo sistema social, es un ente dinámico que dispone y actúa de acuerdo con sus objetivos y sus propios fines, generalmente cambiantes.

4. Para prolongar su existencia, la empresa ha de adaptarse continuamente, y sin cesar, al entorno donde se inserta.

Hemos definido al entorno donde se desenvuelve la empresa como un gran supersistema, formado a su vez por otros subsistemas: político, socio-económico, jurídico, mercado, etc. Por tanto es la organización la que esta inserta en este supersistema y a su vez, participa proporcionalmente en cada uno de los sistemas que forman el supersistema (la empresa concurre a un mercado, tiene implicaciones legales, se relaciona con la comunidad y con los políticos, etc.).

Por tanto será la empresa el ente que tenga que adaptarse a su entorno o si se prefiere, a su medio ambiente. La empresa que ejerce una influencia sobre el entorno, esta en mejores condiciones de competir que aquellas otras que no realizan ninguna acción sobre el mismo. Pero ¿cuales son las acciones que la empresa puede realizar sobre su entorno?. Básicamente, pueden ser de dos tipos:

- Acciones adaptativas: relaciones de la empresa con su entorno, en términos de aceptación y sometimiento a este.

- Acciones modificativas: procesos de actuación de la emperra o relaciones de la empresa sobre su entorno con la intención de modificarlo.

En ambas acciones, la empresa ha de relacionarse con cada subsistema, pero ambas situaciones son, cualitativamente, bien distintas. La actuación del responsable de Relaciones Públicas va encauzada hacia ese segundo ámbito de acciones de la empresa con su entorno, las acciones modificativas. La relaciones con la prensa se deben

Comun. Inf., v. 6, n. 1, p.30-45, jan./jun. 2003 


\section{8}

configurar necesariamente como agentes de cambio de actitudes de la sociedad respecto a la organización.

Hoy más que nunca, las empresas operan en un gigantesco escenario, a la vista de la opinión pública, por lo que su éxito viene determinado no solo por su actuación en el mercado, sino también por lo que los diversos públicos de ese mercado opinan sobre la empresa. Ya hemos visto como las exigencias de información de la empresa son de doble sentido, ya que el conocimiento del entorno y de sus transformaciones se ha convertido en un requisito imprescindible para cualquier empresa moderna y eficientemente gestionada, y porque la información y la comunicación representan, en nuestros días, un valor económico de suma importancia para la sociedad, en términos de una determinada imagen de empresa.

Una de las tareas primordiales del Relaciones Públicas será la de conseguir que los medios de comunicación se acostumbren siempre a contar con él a la hora de solicitar cualquier información, sea del matiz que sea, logrando, además, que las informaciones cedidas sean fiables. Lo que más valora un periodista es la veracidad de la información que se le ofrece, y en el momento en que esta sea requerida 5 .

Si con anterioridad apuntamos el hecho de que la empresa comunica y da una imagen siempre, lo quiera o no, ¿por qué no controlar y coordinar el proceso para que la imagen transmitida a los diferentes públicos sea buena?. El anonimato y la ocultación siempre transmiten un mensaje negativo de la empresa, ya que cuanto mejor se conoce a esta más favorable será su inserción en el entorno más inmediato.

Lo cierto es que en el entrono social, en ese suprasistema, que definimos anteriormente, muchas ideas preconcebidas sobre la comunicación empresarial están comenzando a cambiar:

1. La información económica ha dejado de ser algo para iniciados, y ha abandonado su lenguaje oscurantista para atraer a un público más general.

2. De otro lado, el público cada vez es más exigente y demanda una información de calidad, a la par que demanda cada vez más un mayor conocimiento de su entorno socioeconómico.

3. cada vez es más corriente ver a profesionales de la comunicación al frente de los departamentos de Relaciones Públicas.

Comun. Inf., v. 6, n. 1, p.30-45, jan./jun. 2003 
4. El empresario y la empresa adquieren un papel social cada vez más importante.

La realidad muestra como las empresas más silenciosas y opacas son las que gozan de peor imagen entre los públicos, mientras que las empresas más dinámicas, modernas y prosperas son, a su vez, las empresas con mayor transparencia informativa. se ha comprobado la existencia de tantas imágenes de empresa como de públicos o audiencias impactados por los mensajes intencionales o no intencionales de esta. Esta multipolaridad de imágenes hace que la identidad de la empresa sea muy heterogénea. Esta es una razón lo suficientemente poderosa como para que la empresa emprenda una estrategia de comunicación de cara a influir positivamente en la opinión de sus diferentes públicos. Por tanto, hasta que la compañía no sea capaz de conocer su propia imagen, será incapaz de comunicarla, y mucho menos, de administrarla.

Muchos empresarios confunden la existencia de una política comunicativa de puertas abiertas con el hecho de que se deba informar de todo. Esto no es así, evidentemente. Es necesario informar de todo lo que sea posible, porque esto demuestra a la opinión pública que no hay nada que ocultar. dentro de esta transparencia, también se debe fomentar la actitud, tan poco frecuentada, de que sean los propios empresarios los que atiendan los requerimientos de información de los periodistas, cuando estos así lo exigen, ya que de esta forma, el periodista no tendrá que acudir a segundas o terceras fuentes para contrastar datos, y además, la información será más objetiva y real. Pero además, no solo es necesario informar cuando solo se requiera la información por parte de los medios, sino que es muy conveniente mantener un "goteo informativo" desde la empresa, materializado en unas relaciones continuas y fluidas, con el doble objetivo de dar a conocer las necesidades propias de la empresa y de recibir y atender las demandas o sugerencias de los medios. Por parte de la empresa, es muy poco recomendable ofrecer, solo, aquella información que más le interesa.

Además, la comunicación por parte de la empresa transmite una determinada imagen de la dirección de la misma. La dirección deberá cuidar especialmente dos imágenes: la suya propia, y la imagen corporativa, la imagen institucional de su empresa.

Entre el Relaciones Públicas y los periodistas debe existir una reciprocidad de intereses e interdependencia: ambos actúan como comunicadores. La forma en que trabaja la prensa de cara a las

Comun. Inf., v. 6, n. 1, p.30-45, jan./jun. 2003 
Relaciones Públicas es la siguiente: la función principal de un medio de comunicación es ofrecer noticias a sus lectores. Para conseguir el respeto de la prensa, el Relaciones Públicas deberá ofrecer noticias fidedignas y de buena fe. Considerando que un periodista trabaja contra reloj, no se le puede ofrecer información de escasa relevancia, de lo contrario el Relaciones Públicas desacreditaremos ante el propio periodista y ante su medio de comunicación.

Las relaciones con la prensa han de estar basadas en la honradez. Este es un principio fundamental. Si se dice la verdad a la prensa, esta nunca defraudara a la empresa ${ }^{6}$.

\section{¿Cuando debe informar la organización?}

En una campaña continuada de relaciones con la prensa, una organización debe informar:

1. Por iniciativa propia: siempre que se considere que se ha producido un hecho noticiable, digno de ser conocido por los públicos de la compañía, o bien cuando se deseen expresar opiniones sobre acontecimientos específicos. Este entorno esta cubierto por el Plan de comunicación de la compañía, en el que se establece el planning de noticias, su periodicidad y los medios de comunicación seleccionados.

2. A requerimiento de los medios de comunicación: cuando los medios estimen que la empresa es importante para sus audiencias, entonces se dirigirán a la organización en demanda de informaciones. estos acontecimientos pueden tener la forma de entrevistas, declaraciones, noticias, documentación, cifras, participación en coloquios o debates, ruedas de prensa, etc.

Los medios de comunicación y los periodistas que en ellos trabajan ejercen un doble papel en la sociedad. Por un lado deben adaptarse a las exigencias informativas de cada tiempo y lugar, y así lo requieren sus públicos y sus anunciantes, y por otra parte ejercen una capacidad de critica y un poder importante sobre la masa, gozan de la posibilidad de ejercer de contrapoder, modificando los hábitos, ideas y los valores dominantes en la sociedad, y también actúan como un elemento dinamizador al divulgar nuevas ideas y saberes, aumentando el nivel cultural de los lectores y creando una necesidad de mayores flujos de información.

Comun. Inf., v. 6, n. 1, p.30-45, jan./jun. 2003 
En este sentido, un medio de comunicación desempeña un papel fundamental en la información o desinformación del público, cuando informan justamente, cuando callan o exageran intencionadamente, 0 cuando sugieren, y la opinión pública, a su vez, tiene una gran influencia sobre la marcha de una empresa y sobre su éxito en los mercados.

En realidad, la misión de los medios de comunicación es contar la verdad de los sucesos. pero $¿$ Que verdad? Si consideramos que la información que se le suministra al periodista esta mediatizada profundamente por diferentes factores de índole interna y externa, esa verdad no existe como tal verdad, sino como una dimensión deformada de la verdad absoluta o ideal. Esa verdad deformada es el producto noticioso de los medios de comunicación.

Hemos hablado de una serie de factores de índole interna y externa, a la hora de interpretar los sucesos. Internamente pueden influir sobre el redactor sus prejuicios y su personalidad, la linea editorial del medio para el que trabaja, y su posición y estatus dentro de este. Externamente, la información suministrada al periodista puede sufrir deformaciones procedentes de las interpretaciones de las fuentes, o bien de los grupos de presión que tratan de interferir en la materia prima informativa de cualquier medio de comunicación.

\section{Principales avancesen el mundo actual}

Los cambios tecnológicos y sociales han transformado el papel de las Relaciones Públicas en la década de los 90. La economía global ha propiciado que las Relaciones Públicas abandonen su lugar secundario, que le relegaba a una mera técnica al servicio del marketing, para convertirse en una función directiva. Los avances en el mundo actual están relacionados con los siguientes campos:

- La economía global: la internacionalización de la empresa implica que, tanto las empresas como las Relaciones Públicas tienen que aprender sobre las culturas, practicas comerciales e idiomas extranjeros.

- Calidad del medio ambiente: la preocupación por el medio ambiente es una preocupación general. En muchos países, los ciudadanos creen que la conservación del medio ambiente es una norma tan poderosa o más que cualquier otra norma. A los ciudadanos les preocupa la calidad de vida y la salud, y dado que todas estas cuestiones

Comun. Inf., v. 6, n. 1, p.30-45, jan./jun. 2003 


\section{2}

trascienden el ámbito nacional, el dialogo y la cooperación internacionales constituyen requisitos previos para poder alcanzar una solución efectiva.

- Crecimiento como función gerencial de las empresas: la caída de las ventas, debido a cuestiones medioambientales, ha provocado que las Relaciones Públicas desempeñen un papel fundamental en el proceso de planificación estratégica la formulación de políticas empresariales.

- Nuevo énfasis en la resolución de conflictos (issues management): muchas empresas están intentando resolver cuestiones públicas. Los gobiernos también necesitan la ayuda de expertos en la gestión de conflictos y en la resolución de problemas.

- Proliferación de públicos: la división de los mercados masivos en cientos de pequeños mercados se inició, sobre todo, en la década de 1980 y ha continuado hasta ahora. El personal de Relaciones Públicas ha utilizado la micro demografia (división de públicos en función de la edad, genero, nivel educativo y otras variables análogas). La micro demografía se utiliza para llegar a diversos públicos con información personalizada. Además de los públicos tradicionales como los consumidores o accionistas, las audiencias se han ido fragmentando cada vez más en función de grupos con intereses específicos.

- Declive de los medios de comunicación de masas: la fragmentación de los públicos implica la perdida de peso de los medios de comunicación de masas como vehículos para acceder a las distintas audiencias. La tecnología esta proporcionando muchos caminos para transmitir mensajes a audiencias especificas. Los términos clave son programación por segmentos y selección específica. Los medios masivos ceden terreno frente a los medios especializados, donde los mensajes son personalizadlos en función de audiencias reducidas y bien definidas. Esto significa que hay que comprender a los grupos de interés particulares, trabajar con sus medios y desarrollar una diversidad de mensajes para alcanzar a distintas audiencias.

- Rápido desarrollo de las nuevas tecnologias mediáticas: cada año aumenta el numero de personas que acceden a Internet, y también de aquellas que compran electrónicamente (comercio electrónico). Comun. Inf., v. 6, n. 1, p.30-45, jan./jun. 2003 
- La utilización de medios electrónicos por las Relaciones Públicas: las Relaciones Públicas están utilizando cada vez más los medios electrónicos como principal medio de comunicación con sus públicos y con los propios medios tradicionales. Esto se debe al aumento de los usuarios de Internet, las comunidades virtuales y la cada vez mayor utilización de Internet por los periodistas.

- Una comunicación cada vez más personal: las Relaciones Públicas se están comunicando de una forma cada vez más personal y ya no intentan llegar a audiencias masivas. La tecnología hace que sea posible dividir y subdividir hasta llegar a un punto en el que la comunicación es, casi personal, hasta llegar a destruir por completo el concepto de audiencia masiva.

- Relaciones internacionales con los medios: las conferencias de prensa y las reuniones informativas simultaneas de equipos de profesionales de las Relaciones Públicas en diversos países se retransmiten vía satélite. Las empresas mantienen departamentos de Relaciones Públicas descentralizados en diversos continentes, y están publicando documentos informativos en diversos idiomas.

- Mayor prioridad a la comunicación interna: a finales del siglo XX, el personal de muchas empresas de Relaciones Públicas se ha reducido para satisfacer las demandas de un mercado global y de los públicos de interés (stakeholders) de la organización. Ante el recorte de sus beneficios sociales, la lealtad de los empleados ha decaído drásticamente, lo que provoco que los altos directivos establecieran como alta prioridad las Relaciones Públicas para restaurar y mantener la confianza de los trabajadores.

- El profesional cualificado: el personal de Relaciones Públicas de muchas empresas que actúa a escala global ha mejorado sus competencias profesionales así como su formación, no solo en el aspecto de contenidos: política, finanzas, marketing, sino también en cuanto a habilidades de análisis, gestión y dirección de las estrategias (pensamiento estratégico) ${ }^{7}$.

\section{Conclusiones}

Comun. Inf., v. 6, n. 1, p.30-45, jan./jun. 2003 


\section{4}

La aplicación de los principios de la economía mercantilista a otros ámbitos de la vida los individuos, no estrictamente económicos, acaba transformando muchas de las facetas de las relaciones sociales en las organizaciones. Los principios expresados en toda actividad productiva: la capacidad de innovar, emprender, la eficacia, creatividad y cooperación, así como el derecho a la propiedad privada y la libertad de actuación del individuo, característicos de un sistema económico social y de mercado han impregnado de estos principios a otros campos de actuación del hombre en sociedad, y como hemos afirmado, no exclusivamente económicos.

Esta influencia de los principios de la actividad económica se debe, fundamentalmente, a la actuación de los mecanismos mercantiles como mecanismos rectores de toda acción humana en su globalidad. Uno de los elementos fundamentales y constitutivos de un mercado es la información. La información hace posible que oferentes y demandantes lleguen a alcanzar un acuerdo sobre sus transacciones. La información permite conocer a unos y otros cuales son las características de la otra parte, y en definitiva, la información acaba constituyéndose como la materia prima de los intercambios y las relaciones mercantiles y económicas. Las relaciones económicas se acaban configurando, por tanto, como relaciones eminentemente informativas.

\section{Abstract}

It seems that everything is going to change in the relations between organizations and mass media. Organizations are complex and dynamic in a qualified world where relations and contacts should be greater. The value of an organization is in its values transmited to the market. Society demands a wide range of informations and communications with political and economic interests. Voracity of information also find echo in mass media, more of them included in specialized media.

Key words: Public Relations; Information Society; Specialized Journalism; Public Opinion; Persuasion; Publics; Organizational Communications; Segmentation; Positioning.

\section{Notas y citas}

SOTELO ENRÍQUEZ, C. (2001): Introducción a la comunicación institucional. Ariel Comunicación. Barcelona, p. 9.

PIÑUEL, J.L. y WESTPHALEN, S. (1993): La dirección de comunicación. Ediciones del Prado, Madrid, p. 573 y sig.

Comun. Inf. v. 6, n. 1, p.30-45, jan./jun. 2003 
PIÑUEL y WESTPHALEN, Op cit.

SANZ DE LA TAJADA, L.A. La importancia de la comunicación para la empresa Jornada Sobre RELACIONES DE LA EMPRESA CON LOS MEDIOS DE COMUNICACION. Valladolid. 16 de abril de 1997.

Cit. en MARTíN; F. (1995): Comunicación en empresas e instituciones. De la consultora a la Dirección de Comunicación. Universidad de Salamanca, p. 82.

Cit. en LLOYD, H.; Lloyd, P (1990): Relaciones Públicas. Pirámide, Madrid, pp. $135 \mathrm{y}$ sig.

WILCOX, D. Et al (2000): Relaciones Públicas. Estrategias y tácticas. Addison Wesley, Madrid, pp. 54 y sig.

Comun. Inf., v. 6, n. 1, p.30-45, jan./jun. 2003 